\title{
Connecting breast cancer survivors for exercise: protocol for a two-arm randomized controlled trial
}

\author{
Jenna Smith-Turchyn ${ }^{*}$ (D), Michelle E. McCowan², Erin O'Loughlin², Angela J. Fong ${ }^{3}$, Meghan H. McDonough ${ }^{4}$, \\ Daniel Santa Mina ${ }^{2}$, Kelly P. Arbour-Nicitopoulos², Linda Trinh² ${ }^{2}$, Jennifer M. Jones ${ }^{5}$, Jackie L. Bender ${ }^{5}$, \\ S. Nicole Culos-Reed ${ }^{4}$, Jennifer R. Tomasone ${ }^{6}$, Madison F. Vani ${ }^{2}$ and Catherine M. Sabiston ${ }^{2 *}$
}

\begin{abstract}
Background: Peer-based exercise interventions that cultivate new opportunities for support with a fellow cancer survivor may result in increased exercise volume. It is not clear whether adding qualified exercise professional (QEP) support to peer-based interventions improves health outcomes. Therefore, the purpose of this study is to determine whether breast cancer survivor (BCS) dyads who receive 10 weekly sessions of virtually delivered QEP support have improved outcomes compared to BCS dyads who do not receive QEP support.

Methods: Participants Adult BCS with medical clearance for exercise, who have an internet-connected device, and currently engage in $<150$ min of moderate-intensity exercise per week. Intervention BCS will be matched using evidence-based criteria. The intervention group will receive dyadic exercise information sessions and a program tailored by a QEP for 10 weeks (intervention period) and have access to the QEP for an additional 4 weeks (tapering period). The control will not receive any QEP support. Outcomes The primary outcome is post-intervention selfreported exercise volume. Secondary outcomes include device-assessed exercise volume (i.e., Fitbit), social support, and health-related quality of life. Randomization 108 participants, matched in dyads, will be randomized 1:1 to the MatchQEP or Match groups using a web-based scheme. Statistical analysis Outcomes will be measured at baseline, post-intervention, post-tapering, and at 12 weeks post-intervention follow-up.
\end{abstract}

Discussion: The findings from this RCT will determine if matched BCS dyads who receive 10 weeks of virtually delivered QEP support have higher levels of self-report and device-measured exercise, social support, and health related quality of life compared to matched dyads without QEP-delivered exercise guidance. To our knowledge this will be the first study to assess the combined effect of peer- and QEP support on exercise volume. Project findings will inform and optimize intervention methods aimed to increase exercise among BCS through accessible exercise supports.

Trial Registration: The study is registered on ClinicalTrials.gov (study identifier: NCT04771975, protocol Version Number: 2, date: July 22, 2021).

Keywords: Exercise, Physical activity, Peer-based interventions, Social support, Breast cancer

\footnotetext{
*Correspondence: smithif@mcmaster.ca; catherine.sabiston@utoronto.ca ${ }^{1}$ School of Rehabilitation Science, McMaster University, 1400 Main Street West, Hamilton, ON L8S 1C7, Canada

${ }^{2}$ Faculty of Kinesiology and Physical Education, University of Toronto, Toronto, Canada

Full list of author information is available at the end of the article
}

\section{Background}

One in eight women in Canada will be diagnosed with breast cancer in their lifetime [1]. Despite increasing survival rates [1] and attention to managing the sequelae of breast cancer, survivors face numerous physical and original author(s) and the source, provide a link to the Creative Commons licence, and indicate if changes were made. The images or other third party material in this article are included in the article's Creative Commons licence, unless indicated otherwise in a credit line to the material. If material is not included in the article's Creative Commons licence and your intended use is not permitted by statutory regulation or exceeds the permitted use, you will need to obtain permission directly from the copyright holder. To view a copy of this licence, visit http://creativecommons.org/licenses/by/4.0/. The Creative Commons Public Domain Dedication waiver (http://creativeco mmons.org/publicdomain/zero/1.0/) applies to the data made available in this article, unless otherwise stated in a credit line to the data. 
mental health threats during and after primary treatment that impact their daily functioning and health-related quality of life (HRQOL) [2-5]. Exercise is well-established as a safe and feasible means of improving health and wellbeing through all phases of breast cancer survivorship, including for individuals living with metastatic disease [5-13]. There is long-standing evidence from randomized controlled trials (RCTs) involving breast cancer survivors (BCS) that exercise improves physical, mental, and social health indicators linked specifically to HRQOL; and that exercise is associated with reduced risk of cancer recurrence, cancer-specific mortality, and allcause mortality [5-15]. Cancer exercise guidelines recommend that BCS engage in a minimum of $90-150 \mathrm{~min}$ of at least moderate intensity exercise per week and resistance training at least two times per week [16-18]; however, the majority of BCS do not meet these guidelines [19-22].

In addition to the common environmental and health system exercise barriers that people without cancer face, BCS report that the physical effects of breast cancer treatment pose an additional barrier to exercise [20, 23, 24]. Lack of social support and a lack of access to appropriate exercise programming also prevent engagement in exercise among BCS [20, 23, 25, 26]. For survivors in 'hard to reach' demographic groups (e.g., young adults, those living in rural communities, and/or in an area with low socioeconomic status), finding age-appropriate, affordable, and accessible exercise support is particularly challenging [25].

Social support is positively associated with exercise among BCS [27-29], but few studies have included a systematic examination of the influence of different sources and forms of support within exercise interventions (e.g., professional support vs. spousal support/ caregiver support vs. support from other survivors) [30-32]. The amount and quality of exercise-related support received has focused on either tangible assistance (e.g., people giving you materials or products that help you to exercise), emotional support (e.g., people providing empathy or care when you discuss the difficulties of exercise), informational support (e.g., people providing information on the benefits of exercise), or esteem support (e.g., people providing encouragement that helps you to exercise) [30]. While these different types of support can come from a variety of sources within a breast cancer survivor's social network (e.g., family, friends, colleagues, trusted health professionals, and support group members), peer-to-peer support external to an existing social network has not been studied in depth. Drawing from existing evidence, peer-based interventions that intentionally cultivate new opportunities for support (i.e., receiving support from a peer that is outside of, or in addition to, one's existing support network [33]) can result in positive changes to exercise behavior that can match the effectiveness of professionally delivered supports [34, 35]. Furthermore, peer support interventions that can be delivered remotely have been successful in increasing moderate to vigorous physical activity (MVPA) among BCS [36, 37]. Unfortunately, BCS still report unmet needs related to their ability to find an exercise support peer, especially one who shares the lived experience of cancer [38]. BCS also report challenges with accessing support for exercise from qualified health professionals $[24,25]$.

Online exercise peer matching systems have been developed specifically for women with cancer in an effort to mitigate social support-related barriers to exercise (see ActiveMatch [activematch.ca] and 2Unstoppable [2unstoppable.org]). The peer-to-peer relationship offers unique benefits and is likely to involve different pathways to exercise initiation and maintenance in comparison to professionally delivered exercise support [35]. Nonetheless, even if two survivors are well-matched as exercise peers, this form of peer-to-peer support does not directly address the barrier of a lack of access to exercise guidance from qualified health professionals [39]. Undoubtedly, the relationship between two untrained peers (two survivors) is distinct from the relationship that exists between a survivor and a qualified exercise professional (QEP). As such, the addition of QEP support has the potential to address survivors' desire for tailored exercise guidance and synergistically enhance the benefits of receiving social support from a well-matched exercise peer. Furthermore, exercise guidelines for cancer survivors specify the importance of supervised exercise programming [16-18], and suggest that QEP support may be most beneficial for BCS who are the least active and who have the most to gain from exercise support [40].

In the current study, the primary aim is to examine the independent effects of matched peers on self-reported exercise volume among BCS in a RCT with one group of dyads assigned to receive QEP support and the other group of BCS dyads not receiving QEP support. Secondary aims include exploring the effects of matched peers on device-measured moderate-vigorous physical activity (e.g., Fitbit), perceived social support, and HRQOL. Actor-Partner Interdependence models (APIM) (Fig. 1) will be used as a framework to study the peer (dyadic/ partner) effects and the effects of the intervention (QEP support) over time [41-43]. Also, a strategic assessment of the cost-effectiveness of offering virtually delivered QEP sessions to matched BCS dyads will help to inform and optimize methods of increasing exercise among BCS through accessible exercise supports. 


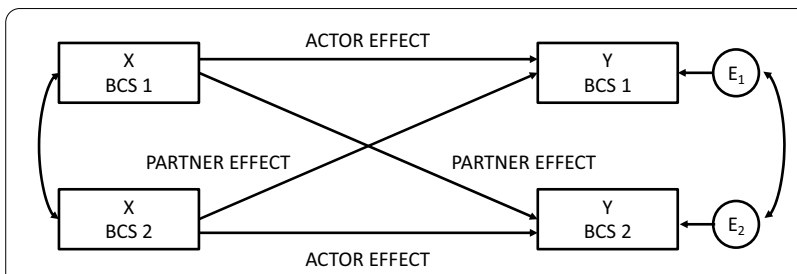

Fig. 1 Actor-Partner Interdependence Model framework for indistinguishable dyads testing the effects of breast cancer survivor peers on moderate-to-vigorous physical activity ( $X=$ Baseline, $Y=$ Post-10 week intervention as primary endpoint)

\section{Study purpose}

The purpose of this study is to examine interpersonal and individual effects of partnering $\mathrm{BCS}$ with a peer and a QEP on self-reported exercise volume (MVPA; primary outcome), and device-measured exercise volume (MVPA; Fitbit), social support, and HRQOL (secondary outcomes). Cost-effectiveness and intervention adherence will also be explored as tertiary outcomes. The effects of a peer and QEP-support intervention group, labelled MatchQEP, will be compared to a control group of BCS who are matched with a peer, but not a QEP, labeled Match.

There are three main research questions and hypotheses (also presented in Table 1):

1. Are there any differences between the MatchQEP group and the control group on self-reported MVPA after the intervention (10 weeks post baseline)? Based on the consistent evidence for positive effects of exercise specialists on MVPA, we expect the peers in the MatchQEP group to report greater increases in MVPA than the peers in the control group at postintervention. We expect these differences to be maintained at post-tapering and follow-up data collections.

2. Does exercise peer 1's exercise volume (minutes of MVPA) at baseline predict exericse peer 2's exercise volume after the intervention (and vice versa)? A positive peer effect between baseline and post-intervention within the MatchQEP group is expected as the QEP is expected to work with the dyads in ways to support each other's MVPA. In contrast, nonsignificant or small peer effects are expected to be observed within the control group who receive no QEP support.

3. To what extent does MVPA at baseline predict MVPA after the intervention? The QEP is expected to improve MVPA and the effects are likely to be strongest for the most inactive women. Therefore, the stability of individual differences in MVPA may be lower in the group that has QEP support compared to the control group.

All research questions also align with the secondary outcomes, such that device (e.g., Fitbit) measured MVPA, social support, and HRQOL will be higher among the MatchQEP group.

Table 1 Research questions and hypotheses for the main study effects exploring the impact of partner (i.e., peer) effects and qualified exercise professional (QEP) support on physical activity among breast cancer survivors

\begin{tabular}{|c|c|c|}
\hline Type of effects & Explanation & Hypotheses \\
\hline \multicolumn{3}{|l|}{ Interpersonal effects } \\
\hline Partner effect (P1-P2) & Influence of the peer $1(\mathrm{P} 1)^{\prime}$ s baseline MVPA on the peer $2(\mathrm{P} 2)$ 's post-intervention MVPA & $\begin{array}{l}\text { Positive effect in intervention } \\
\text { group, no effect in control group }\end{array}$ \\
\hline partner effect (P2-P1) & Influence of the P2's baseline MVPA on the P1's post-intervention MVPA & $\begin{array}{l}\text { Positive effect in intervention } \\
\text { group, no effect in control group }\end{array}$ \\
\hline \multicolumn{3}{|l|}{ Individual effects } \\
\hline Actor effect $_{(\mathrm{P} 1)}$ & Stability of the P1's MVPA from baseline to post-intervention & Intervention group $<$ control group \\
\hline Actor effect $_{(\mathrm{P} 2)}$ & Stability of the P2's MVPA from baseline to post-intervention & Intervention group $<$ control group \\
\hline Treatment effect $(\mathrm{P} 1)$ & $\begin{array}{l}\text { Difference between the P1's mean post-intervention MVPA of the intervention group and of } \\
\text { the control group }\end{array}$ & Intervention group > control group \\
\hline Treatment effect (P2) & $\begin{array}{l}\text { Difference between the P2's mean post-intervention MVPA of the intervention group and of } \\
\text { the control group }\end{array}$ & Intervention group > control group \\
\hline \multicolumn{3}{|l|}{ Intervention effects } \\
\hline Adherence & $\begin{array}{l}\text { Adherence is established if BCS in the MatchQEP group attend at least } 7 \text { out of } 10 \text { assigned } \\
\text { sessions with the QEP } \\
\text { Adherence to peer support in both MatchQEP and Match groups will be established based } \\
\text { on the number of times the peers connect (expecting at least once per week for } 10 \text { weeks) }\end{array}$ & Adherence will be established \\
\hline Cost-effectiveness & $\begin{array}{l}\text { Direct costs will be compared between the MatchQEP and Match groups and between the } \\
\text { MatchQEP group to traditional QEP services in Canada }\end{array}$ & MatchQEP will be cost-effective \\
\hline
\end{tabular}


Intervention adherence and cost-effectiveness will also be explored. We expect BCS to adhere to the MatchQEP group by attending at least $70 \%$ of the sessions $[44,45]$ and BCS to adhere to the Match group by connecting with their partners at least once per week for 10 weeks. We expect the MatchQEP group to be cost-effective (e.g., have lower direct costs compared to traditional in person and clinical QEP services in Canada and a favorable incremental cost-effectiveness ratio compared to the Match group). The MatchQEP group is also expected to report less health care use (e.g., health care facility visits, doctor visits, procedures received, support services used, and loss of work) compared to the Match group.

\section{Methods}

\section{Study design and participants}

This is a two-arm RCT with validated outcome assessment measures. The protocol adheres to the CONSORT guidelines (Fig. 2) [46] and SPIRIT recommendations for reporting of clinical trial protocols [47]. Eligible participants are: (1) English-speaking female BCS; (2) diagnosed with primary stage $0-I V$ breast cancer, at any stage of treatment; (3) living in Canada; (4) aged 18 years or older; (5) medically cleared for exercise; (6) connected to the internet using any device (e.g., computer, tablet or smartphone; preferably with webcam); and (7) currently engaging in less than $150 \mathrm{~min}$ of MVPA per week [1618]. Participants will be excluded from the study if they report any contraindications to exercise, or had recent or have planned surgery of any kind (including reconstructive surgery).

\section{Recruitment}

BCS will be recruited using digital recruitment materials. Study investigators will send a recruitment email and social media posts to select community-based cancer programs, services, and organizations across Canada for administrators at these organizations to share with their community members. All recruitment materials will include instructions for interested individuals to contact the study team directly. Eligibility will be confirmed by email or phone call by a member of the study team and all eligible participants will provide informed consent prior to enrollment. The University of Toronto's Human Research Ethics Unit has approved this study (protocol \#00038665).

\section{Sample size}

Sample size calculation was carried out based on APIM power analysis for indistinguishable dyads [48]. Specifically, the alpha was set to 0.05 and the sample size estimate is the smallest number of dyads required to detect the effect when power is at least 0.8. Based on standardized regression coefficient effect size estimates (beta; $\beta$ ) drawn from a pilot study of 46 women matched for exercise (23 dyads) assessed over 21 days (i.e., reflecting the Match group in the current study), the actor effect size was $\beta=0.33$, the partner effect size was $\beta=0.28$, and the correlation between the two women's self-report scores on minutes of exercise at baseline was $r=0.46$. The correlation of the errors was set at 0.30 . At minimum, 32 total dyads are needed to have adequate power to detect an actor effect and 43 total dyads are needed to have adequate power to detect a partner effect for a required sample size of $N=86$. Attrition may be $30 \%$ [49], and 11 additional dyads will be recruited for a total sample size of 108 women (54 dyads total across both groups).

\section{Treatment allocation and randomization}

Dyads will be randomized (1:1) to the intervention (MatchQEP) or control (Match) group prior to baseline assessment. Treatment allocation will be centrally randomized using a web-based scheme (https://www.rando mizer.org/). The randomization will be completed by a PhD student external to the research team and group allocation will be assigned in the order in which participants have completed an initial background and demographics questionnaire and have been matched with a peer for the study. Although the research coordinator(s), QEPs, and participants cannot be blinded to treatment allocation, the primary investigators and statistician completing data analysis will be blinded.

\section{Intervention}

Matching of participants into dyads will be conducted by a research coordinator based on evidence-informed criteria developed in our formative study [50]. For participants to be matched, they must: (1) live in the same time zone, (2) be in the same age range (i.e., within \pm 10 years of age), and (3) self-report similar current exercise volume (i.e., within $30 \mathrm{~min}$ per week). Beyond these core matching criteria, we will create discrepancy scores based on other personal and cancer-related characteristics, such as cancer stage at diagnosis, treatment status, time since treatment, participants' family situation (e.g., having a romantic partner and/or children), and exercise preferences (e.g., preferred time of day for exercise) [25, 51] and account for discrepancies in these characteristics in analyses. All participants will receive an 'Exercise Peer Support Guide' that provides tips and strategies for supporting their exercise peer $[52,53]$ and a one-page infographic highlighting current exercise guidelines for cancer survivors [16-18]. All participants will be given a Fitbit wearable activity tracking device, which will be used for device-measured MVPA. Matched exercise peers will be introduced to one another by a research 


\begin{tabular}{|c|c|c|c|}
\hline Participant Recruitment & \multicolumn{2}{|c|}{$\begin{array}{l}\text { Participants recruited based on inclusion criteria: female breast cancer } \\
\text { survivors, English-speaking, living in Canada, } \geq 18 \text { years, cleared for } \\
\text { exercise (by QEP, with physician clearance as needed), has an internet- } \\
\text { connected device, engaging in }<150 \text { minutes of at least moderate } \\
\text { intensity exercise per week }\end{array}$} & \\
\hline Screening & \multicolumn{2}{|c|}{$\begin{array}{l}\text { Potential participants will be contacted by a member of the research } \\
\text { team to discuss study objectives, eligibility, and potential enrollment }\end{array}$} & \\
\hline Enrollment \& Matching & \multicolumn{2}{|c|}{$\begin{array}{l}\text { Eligible participants will be invited to complete the study consent form, } \\
\text { be mailed a Fitbit, and complete a demographics questionnaire, } \\
\text { including questions related to partner matching criteria. Participants } \\
\text { will be matched (dyads created) by a member of the research team } \\
\text { based on similarity in time zone, age, current exercise, and other } \\
\text { personal and cancer-related characteristics }\end{array}$} & \\
\hline Randomization & $\frac{\text { MatchQEP Group }}{\text { ( } n=27 \text { dyads })}$ & $\frac{\text { Match Group }}{(n=27 \text { dyads) }}$ & \\
\hline Baseline Assessment & \multicolumn{2}{|c|}{$\begin{array}{l}\text { Outcome measures assessed in both groups: Godin Leisure Time } \\
\text { Exercise Questionnaire, Fitbit data, Sedentary Behavior Questionnaire, } \\
\text { Social Support Survey questions, SF-12, EQ-5D }\end{array}$} & \\
\hline \multirow[b]{2}{*}{$\begin{array}{r}\text { Post-Intervention Assessment } \\
\text { (10 weeks post-baseline) }\end{array}$} & $\begin{array}{l}\text { MatchQEP Group: } \\
\text { Participants introduced to } \\
\text { partner, given 'exercise partner } \\
\text { support guide' and exercise } \\
\text { guidelines for cancer survivors, } \\
\text { and meet (as a dyad) with QEP } \\
\text { weekly over Zoom for } 10 \text { weeks }\end{array}$ & $\begin{array}{l}\text { Match Group: } \\
\text { Participants introduced to } \\
\text { partner and given 'exercise } \\
\text { partner support guide' and } \\
\text { exercise guidelines for cancer } \\
\text { survivors (no QEP support) } \\
\end{array}$ & \\
\hline & \multicolumn{2}{|c|}{$\begin{array}{c}\text { Outcome measures assessed in both groups: Fitbit data, Godin Leisure } \\
\text { Time Exercise Questionnaire, Sedentary Behavior Questionnaire, Social } \\
\text { Support Survey questions, SF-12, EQ-5D, Use of health care services } \\
\text { questionnaire. The WAI-SR will also be completed at this time for the } \\
\text { MatchQEP group only }\end{array}$} & $\begin{array}{l}\text { Effectiveness of } \\
\text { intervention on each } \\
\text { outcome will be } \\
\text { determined }\end{array}$ \\
\hline Tapering Period & $\begin{array}{l}\text { MatchQEP Group: } \\
\text { Participants given access to QEP } \\
\text { consultation as needed, by } \\
\text { request (tracked), for } 4 \text { weeks } \\
\text { after intervention end }\end{array}$ & $\begin{array}{l}\text { Match Group: } \\
\text { No QEP support }\end{array}$ & \\
\hline $\begin{array}{l}\text { Post-Tapering Assessment } \\
\text { (14 weeks post-baseline) }\end{array}$ & \multicolumn{2}{|c|}{$\begin{array}{c}\text { Outcome measures assessed in both groups: Fitbit data, Godin Leisure } \\
\text { Time Exercise Questionnaire, Sedentary Behavior Questionnaire, Social } \\
\text { Support Survey questions, SF-12, EQ-5D, Use of health care services } \\
\text { questionnaire. The WAI-SR will also be completed at this time for the } \\
\text { MatchQEP group only }\end{array}$} & $\begin{array}{l}\text { Effectiveness of } \\
\text { intervention on each } \\
\text { outcome will be } \\
\text { determined }\end{array}$ \\
\hline $\begin{array}{r}\text { 12-week Follow-Up Assessment } \\
\text { (26 weeks post-baseline) }\end{array}$ & $\begin{array}{r}\text { Outcome measures assessed in b } \\
\text { Time Exercise Questionnaire, Sed } \\
\text { Support Survey questions, SF-12 } \\
\text { ques }\end{array}$ & $\begin{array}{l}\text { Dups: Fitbit data, Godin Leisure } \\
\text { Behavior Questionnaire, Social } \\
\text { D, Use of health care services } \\
\text { ire }\end{array}$ & $\begin{array}{l}\text { Effectiveness of } \\
\text { intervention on each } \\
\text { outcome will be } \\
\text { determined }\end{array}$ \\
\hline \multicolumn{3}{|l|}{ Fig. 2 Study flow } & \\
\hline
\end{tabular}

assistant via Zoom (video conferencing platform) prior to the start of the study period. Refer to Fig. 2 for a study flow chart.

\section{Intervention condition}

Participants in the MatchQEP group will receive exercise information and program sessions tailored by a QEP specifically for each BCS in the dyad. A single QEP, with advanced training on exercise for individuals with cancer, will provide all QEP sessions for each dyad. The study QEP also has research experience leading longitudinal and experimental trials while also holding a $\mathrm{PhD}$ in behavioral medicine. MatchQEP dyads will meet with the QEP via Zoom once per week for 10 weeks. Each weekly session will last up to $60 \mathrm{~min}$ and will include a review of the exercise program, achievement of goals/ action plans set, adverse effects/events, and discussion on overcoming barriers to completing the prescribed exercise program. The actual length of the session, in minutes, will be noted. Additionally, the QEP will 
follow a standardized guide to deliver specific sessions each week based on behavior change counselling support strategies [54, 55] (see "Appendix"). Based on current exercise recommendations for cancer survivors [16-18], the overall goal of the QEP-prescribed exercise intervention is for each member of the dyad to complete a minimum of 150 min of MVPA per week, which may include both aerobic and resistance forms. Consistent with home-based exercise strategies, the QEP will tailor the program based on personal circumstances, including cancer-related characteristics, side effects, current fitness level, and available space and equipment [16]. MatchQEP group participants will have the opportunity to reach out to the QEP individually if they need to ask specific and personal questions during the intervention period. For 4 weeks following the completion of the 10 -week intervention, the QEP will be available for consultation (i.e., a post-intervention tapering period) as needed by the MatchQEP group participants. This tapering period is important for understanding strategies to successfully taper BCS from exercise trials. All interactions between the QEP and the dyads from intervention initiation until the end of the tapering period will be recorded.

\section{Control condition}

BCS in the Match (control) group will independently communicate and support each other around exercise for 10 weeks. They will not have any contact with a QEP during this time and will structure their own communication with their matched peer. After the 12 weeks post-intervention follow-up assessment (26 weeks post-baseline), BCS in this group will be offered a single consultation session with a QEP to discuss exercise-related questions.

\section{Data collection and measures}

Following informed consent, all BCS will complete a demographics questionnaire, including questions related to peer matching criteria, treatment, and exercise history. To determine the effectiveness of the intervention, BCS will complete exercise volume, social support, and HRQOL questionnaires online at four time points: baseline, post 10-week intervention, post 4-week tapering (i.e., 14 weeks post-baseline), and at 12 weeks post-intervention follow-up (i.e., 26 weeks post-baseline). BCS will be asked to wear the Fitbit to collect device-measured MVPA for one week at all four timepoints. Self-reported sedentary behavior will be assessed as a potential covariate at all time points. The QEP alliance (i.e., connection to the QEP support provider) will be assessed as a covariate at post-intervention and post-tapering time points. Adherence to the intervention will be calculated at the end of the intervention period (10 weeks) and cost-effectiveness will be assessed at post-intervention (10 weeks), post-tapering (14 weeks), and at the 12-week post-intervention follow-up (26 weeks after baseline assessment).

\section{Primary outcome}

Exercise volume at the post 10 -week intervention time point, will be measured using a valid and reliable selfreport survey (modified Godin Leisure Time Exercise Questionnaire) [56-58]. The modified Godin Leisure Time Exercise Questionnaire is a self-report measure which asks respondents to give weekly frequencies and durations of strenuous, moderate, and mild aerobic activities. Resistance training volume will also be selfreported using the same format as the aerobic activities (e.g., frequency and time metrics). Responses on moderate and strenuous activities are summed to determine total MVPA volume. This measure will also be assessed at the post-tapering (i.e., 14-weeks) and 12 weeks postintervention follow-up (i.e., 26 weeks post-baseline) time points.

\section{Secondary outcomes}

Device-measured MVPA, social support, and HRQOL are secondary outcomes.

\section{Device-measured MVPA}

Exercise volume will also be assessed using a tracking device (Fitbit Inspire ${ }^{\circledR} 2$ accelerometer). Adherence to Fitbit's use in cancer survivors is high $[59,60]$ and Fitbit exercise data has demonstrated high correlation to Actigraph measures in this population [59]. Fitbit devices will be mailed to BCS at study inception and will be required to be worn for 7 consecutive days during the four primary data collections to determine their average daily and weekly

s of MVPA and step count. BCS are not required to wear the device outside of the data collection timeframes, but can wear them if they choose. Total wear time and corresponding data will be collected by a research assistant via the online Fitbit database in each participants unique, deidentified study Fitbit account and will be used to characterize the use of Fitbit devices over the entire study duration. Participants will keep the devices post-intervention.

\section{Social support}

A breast cancer-specific version of the Social Support Survey (SSS) [31, 61, 62] will be used to assess seven dimensions of social support: listening support, task challenge, emotional support, esteem support, reality confirmation, tangible assistance, and understanding breast cancer support [31, 61, 62]. Within each dimension, respondents will be asked to score two questions on a five-point Likert scale (amount (total number) and 
satisfaction with each type of support rated from very dissatisfied to very satisfied). The SSS has demonstrated adequate construct validity in sport settings [57] and has been validated in a sample of BCS [63]. Participants will also rate the amount (total number) and perceived quality (from very dissatisfied to very satisfied) of support they receive in the following exercise-related support categories: listening support, task challenge, emotional support, esteem support, reality confirmation, tangible assistance, and informational support. At the post-intervention, post-tapering, and follow-up assessments, participants will rate, on a seven-point scale ranging from 1 (none at all) to 7 (a lot), the amount of each type of exercise-related support that they received from their study exercise partner since the last assessment time. For the purpose of this study, a total social support latent variable will be estimated to account for general and exercisespecific social support. See Additional file 1.

\section{HRQOL}

The Short-Form-12 (SF-12) [64, 65] and the EQ-5D-3L [66] will be used to assess HRQOL. The SF-12 is a selfadministered questionnaire consisting of 12 items addressing eight domains of health (physical functioning, role-physical, bodily pain, general health, vitality, social functioning, role-emotional, and mental health). The SF-12 has demonstrated validity in adult populations when compared to the SF-36, with high correlations for both the physical $(r=0.94-0.96)$ and mental $(r=0.94-$ $0.97)$ domains, showing that it is a practical alternative to the longer SF-36 [65]. The EQ-5D-3L [66] is a two-part measure. The first part using a three-level scale to assess five dimensions of health (mobility, self-care, usual activities, pain/discomfort, and anxiety/depression). The second part assesses responder's perception of their health on a visual analogue scale from 0 (worst imaginable health) to 10 (best imaginable health). This measure is reliable and valid for individuals with cancer [67]. For the purpose of this study, a total latent variable for HRQOL will be tested and used in the main analysis exploring HRQOL effects as a secondary outcome of the study.

\section{Tertiary outcomes}

\section{Intervention adherence}

The QEP will track MatchQEP group adherence by completing a weekly session log. The QEP will record attendance of each BCS at the virtual QEP session as well as whether each individual completed the goals set from the previous session. MatchQEP group adherence is defined as the number of sessions attended divided by the total number of sessions. Adherence in the Match group will be determined by the number of times the peers connect during the 10-week intervention period.

\section{Cost-effectiveness}

The costs of the MatchQEP intervention will be calculated and compared to traditional face-to-face costs of QEP services in Canada and to the Match group alone. Refer to Table 2 for a description of costs to be compared between MatchQEP group and traditional QEP services. The use of health care resources will also be compared between the two groups at the post-intervention, post-tapering, and follow-up time points using a piloted questionnaire [68] that assesses health care facility visits, doctor visits, procedures received, support services used, and loss of work (see Additional file 2).

\section{Descriptive data and covariates}

Age in years, stage of cancer, time since treatment, time since diagnosis, current medications, and exercise preferences will be self-reported at baseline in an online demographic questionnaire. The Sedentary Behavior Questionnaire [69] will be used to assess the amount of time (in hours) per week spent engaged in sedentary pursuits (e.g., watching television, sitting and reading). The scale has demonstrated reliability and validity in adult populations [69]. Sedentary behavior will be a covariate [70]. Exercise peer match quality (assessed on a 5-point scale from 1: very poor match to 5: very good match), amount of time spent with peer, method of connecting with peer, and perceived similarities with peer (assessed on a 5-point scale from not at all similar to extremely similar) will be collected post-intervention and used as covariates in analyses (see Additional file 3). Therapeutic alliance between participants and the QEP will be assessed by each participant in the MatchQEP group at the post intervention (10 week) and post tapering (14 week) time points using the modified Work Alliance Inventory-Short Form Revised (WAI-SR) [71]. The WAI-SR is a twelve-item scale evaluating three domains of therapeutic alliance: (1) agreement between patient and therapist on the goals of treatment, (2) agreement between patient and therapist about the tasks to achieve these goals, and (3) the quality of the bond between the patient and therapist (QEP) [71]. Each item is assessed on a 7-point Likert scale from 0 (never) to 7 (always), with higher scores representing higher satisfaction with the therapist (QEP)-patient relationship [65]. The WAI-SR is a reliable and valid outcome for assessing therapeutic alliance (internal consistency: $\alpha=0.91$, test-retest reliability: $r=0.93$; and construct validity: $r=0.74$ ) [71].

\section{Safety/adverse events}

While exercise is known to be safe for BCS when performed correctly $[14,15]$, minor injuries have the potential to occur in any exercise intervention. To enhance participant safety in this study, all participants will be 
Table 2 Cost of MatchQEP group versus traditional QEP services

$\begin{array}{ll}\begin{array}{ll}\text { MatchQEP group } \\ \text { Program costs }\end{array} & \text { Professional time/labor } \\ & \text { Equipment } \\ \begin{array}{l}\text { Traditional QEP services } \\ \text { Session costs }\end{array} & \\ & \text { Total cost of QEP session } \\ \text { Travel } & \text { Labor } \\ & \text { Mileage estimates } \\ & \text { Public transportation costs } \\ \text { Home-based equipment } & \text { Parking costs }\end{array}$

Consumable materials

Photocopies of home exercises

Printed letters/folders given to survivors

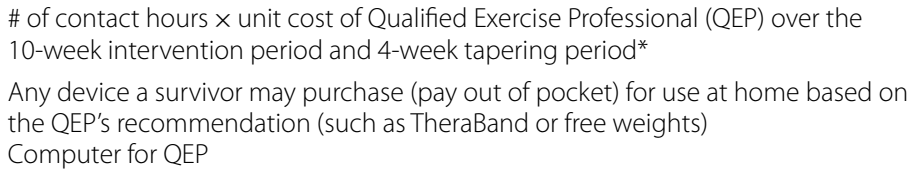
the QEP's recommendation (such as TheraBand or free weights) Computer for QEP

Includes venue use/cost of membership, equipment at venue, preparation time for the QEP

\# of QEP sessions attended during a comparable 10-week period $\times$ cost of each session

Based on distance $(\mathrm{km})$ from an individual's home to the session venue and the cost of gas $(/ \mathrm{km})$

Any form of device that a survivor may purchase for use at home based on the QEP's recommendations (for example, TheraBand or free weights)

\footnotetext{
* For each 1-h contact cost, $1.5 \mathrm{~h}$ of labor will be allocated to account for the preparation time required for each session

\# The cost of training staff will be excluded as staff will already be qualified for their role
}

cleared to exercise prior to enrollment using the PAR$\mathrm{Q}+/ \mathrm{ePARmed}-\mathrm{X}+$, [72] with physician clearance as needed. For the women in the MatchQEP group, tailored exercise recommendations will be provided during the exercise program delivery. Any injuries or side effects that occur in the MatchQEP group will be tracked by the QEP on an 'events' log with all adverse events classified on severity with specific types/class using the "Common Terminology Criteria for Adverse Events". Adverse events will be reported to the Principal Investigator immediately for follow-up with BCS and connection to physician for further consultation.

\section{Statistical analysis}

All analyses will be conducted using R [73] and MPlus 7.4 [74]. Significance will be established at $p<0.05$ for all coefficients. Descriptive statistics (means, frequencies, zero-order correlations) will be computed for all measures over time. The main analysis will be guided by APIM [41] to explore interpersonal and individual effects of the intervention (QEP support) for the primary and secondary outcomes over time.

In the APIM (see Fig. 1), effects of both dyad members are assessed simultaneously by estimating interpersonal peer effects (labelled partner effects in APIM) and individual effects (labelled actor effects in APIM). Three types of effects can be used to explore the research questions examining MVPA among BCS. First, the peer (i.e., partner) effects indicate the average influence of each BCS's MVPA in the dyad. Second, the actor effects assess the average stability of MVPA across the intervention for each dyad member. Specifically, actor effects provide an indication to the effectiveness of the QEP support intervention since MVPA is assumed to be altered by QEP support more strongly when compared to the control group. Third, the average intervention effects will be tested and compared to post-intervention MVPA. The effectiveness of matching BCS dyads on device-measured MVPA, social support, and HRQOL will also be explored in separate models testing actor and partner effects on these secondary outcomes.

Multiple-group structural equation modeling (SEM) will be used to estimate and test an APIM with two groups (MatchQEP vs. Match). To address research question 1, the mean post-intervention levels of MVPA (controlling for actor and partner effects) of the two groups will be compared. For research question 2, the average partner (i.e., peer) effects of MVPA from each BCS in the dyad will be estimated within each group [43]. The effects will be tested by comparing constrained and unconstrained models on chi-square goodness-of-fit values. For research question 3, the average actor effects for MVPA among BCS will be estimated for the MatchQEP and Match groups and will be tested against each other. These analyses will be repeated for device-measured MVPA, social support, and quality of life (secondary outcomes). Measured covariates (e.g., cancer and personal characteristics, partner characteristics and quality of match, therapeutic alliance) will be included in the models as necessary based on their association with the outcomes. 
To assess the intervention effects (tertiary outcomes), adherence will be examined based on the number of sessions attended with the QEP in the MatchQEP group and on the frequency the peers connected over the duration of the study for both MatchQEP and Match participants. Cost-effectiveness will be determined by comparing the direct intervention costs between study groups and between the MatchQEP group and traditional, in-person QEP services. Additionally, cost-effectiveness of the intervention group, compared to control group, will be calculated based on the intervention costs per participant with respect to changes in the assessed health outcomes. Incremental cost-effectiveness ratios will determine the cost of one unit of change for the investigated outcomes. Mean costs of each category of health resource use (health care facility visits, doctor visits, procedures received, support services used) and loss of work will be calculated for each group and compared for significant differences using $t$ tests. All costs will be determined based on current 2021 Ontario Health Care standards in Canadian dollars.

\section{Discussion}

The benefits of exercise for BCS span physical, psychological, and social health [5-11]. Regular exercise also has the potential to decrease mortality in BCS [12-14]. Due to the low rates of health enhancing MVPA among BCS [19-21], efforts are needed to help BCS begin and sustain optimal activity levels. Currently, BCS report a lack of support for exercise and lack of information from a QEP as barriers to exercise participation [20,23]. Tailored exercise information from a QEP and social support, specifically from fellow survivors, are common requests from BCS in order to facilitate engagement in exercise at various points in the survivorship trajectory [23, 75]. The findings from this RCT will determine if matched BCS dyads who receive 10 weeks of virtually delivered QEP support have higher levels of self-report and devicemeasured exercise, social support, and HRQOL compared to matched dyads without QEP-delivered exercise guidance.

Exercise interventions for cancer survivors led by a QEP, and using social support through matched dyads to facilitate exercise, have been separately documented in the literature [76-79]. To our knowledge this will be the first study to assess the combined effect of these two facilitators on exercise. Another unique component of this intervention is that the QEP support provided to the intervention group will be delivered virtually. There are numerous anticipated benefits to this intervention delivery method. First, the virtual delivery of exercise support is thought to meet the needs of diverse populations of cancer survivors, including those who are considered hard to reach (such as those who live in rural/remote areas and young adult cancer survivors) [25, 80]. For example, survivors living in rural areas often do not have access to a QEP trained in cancer rehabilitation and do not have a large community of cancer survivors to get support from and share experiences with [25, 81, 82]. Furthermore, young adult cancer survivors report difficulty accessing exercise services due to the timing and location of available exercise services which are not ideal due to their work and family commitments [25, 83]. Providing virtual delivery of exercise programming will make exercise services more available for many BCS as they will be able to access the intervention from anywhere, at more convenient times. However, since there will be cancer survivors who will not be able to access the internet, future work must consider the issue of technology equity and develop strategies to ensure equitable exerciserelated services for this subgroup of survivors.

Virtual delivery of exercise support also improves the ability to match BCS dyads. Not having to limit matched dyads to those who live in the same geographical area is likely to lead to more well-matched dyads (i.e., matches will include those who have similar personal and cancer-related characteristics). This is important as survivors report needing exercise programs to include those of similar age and fitness level to motivate participation [25]. Furthermore, from a behavioral change perspective, support from similar others has been found to correlate with higher levels of exercise intention and behavior in cancer survivors [84]. Finally, delivering interventions virtually is vital in order to allow exercise services to continue safely during a global pandemic [85]. Currently, few virtually-delivered exercise interventions for cancer survivors are described in the literature [76, 86-88]. While the COVID-19 pandemic has been the impetus for clinicians to quickly change the way they practice, many components of these new healthcare delivery formats will likely continue post-pandemic. Therefore, it is important to evaluate these forms of virtual interventions.

This study will also examine the cost-effectiveness of the virtual QEP intervention for BCS dyads compared to traditional in-person QEP services, as well as a partnerbased intervention without QEP support. Not only will these results have immediate implications for existing dyadic exercise support programs (such as ActiveMatch [activematch.ca] and 2Unstoppable [2unstoppable.org]), but together, the findings will help to determine the most cost-effective way to deliver exercise services to cancer survivors. This information is important to convey to healthcare policy makers as QEPs, clinicians, and researchers strive to make exercise a standard component of cancer care, in a healthcare system with limited funds and space [89]. 


\section{Limitations}

Potential limitations should be taken into account when considering the prospective impact of this study. Specifically, all participants in this study will be English-speaking self-identified female BCS living in Canada. This limits the generalizability of the findings to survivors of other forms of cancer, male and intersex survivors, and non-Anglophones. This lack of generalizability is important to consider as exercise preferences are known to differ based on cancer type and sex. Also, the nature of distance-based interventions excludes those who do not have access to reliable internet and/or an internet-connected device, which may limit the usability of these findings for those individuals.

\section{Appendix: MatchQEP weekly discussion topics}

\begin{tabular}{|c|c|c|}
\hline Week & Topic & $\begin{array}{l}\text { Actions/discussion } \\
\text { points }\end{array}$ \\
\hline 1 & Getting started & $\begin{array}{l}\text { Exercise benefits } \\
\text { Exercise guidelines } \\
\text { Weekly exercise plan/ } \\
\text { schedule/introduce } \\
\text { tracking exercise } \\
\text { Introduce the RPE scale }\end{array}$ \\
\hline 2 & Goal setting & $\begin{array}{l}\text { The "5 why's" exercise } \\
\text { Goal setting and action } \\
\text { planning } \\
\text { F.I.T.T/A.I.M }\end{array}$ \\
\hline 3 & Barriers & $\begin{array}{l}\text { Barriers and obstacles } \\
\text { Solutions } \\
\text { If-then exercise }\end{array}$ \\
\hline 4 & Social support & $\begin{array}{l}\text { Connection } \\
\text { Assess communica- } \\
\text { tion (what works/what } \\
\text { doesn't work) } \\
\text { Types of support and } \\
\text { strategies }\end{array}$ \\
\hline 5 & Self-talk & $\begin{array}{l}\text { All or nothing mindset } \\
\text { Self-compassion state- } \\
\text { ments } \\
\text { Self-talk and mindful- } \\
\text { ness }\end{array}$ \\
\hline 6 & $\begin{array}{l}\text { Importance of recov- } \\
\text { ery and rest }\end{array}$ & $\begin{array}{l}\text { Yoga and stretching } \\
\text { Sleep guidelines } \\
\text { How to recognize stress } \\
\text { and struggling }\end{array}$ \\
\hline 7 & Habit forming & $\begin{array}{l}\text { Habit stacking } \\
\text { Increasing/decreasing } \\
\text { friction } \\
\text { Triggers and cues }\end{array}$ \\
\hline 8 & How to progress & $\begin{array}{l}\text { Exercise progression } \\
\text { strategies } \\
\text { Exercise principles } \\
\text { (adaptation, progressive } \\
\text { overlad, etc.) } \\
\text { How to increase daily } \\
\text { activity (NEAT) } \\
\text { Maintenance is progress }\end{array}$ \\
\hline
\end{tabular}

\begin{tabular}{lll}
\hline Week & Topic & $\begin{array}{l}\text { Actions/discussion } \\
\text { points }\end{array}$ \\
\hline 9 & Maintaining motiva- & Reflect on motivation \\
tion & $\begin{array}{l}\text { Future goal planning } \\
\text { Short, medium, long } \\
\text { term goals (post QEP } \\
\text { sessions) } \\
\end{array}$ & Comeback plan \\
\hline
\end{tabular}

RPE rated of perceived exertion, F.I.T.T frequency, intensity, time, type, A.I.M accoutability, important, and measureable, NEAT non-exercise activity thermogenesis, QEP qualified exercise professional.

\section{Abbreviations}

QEP: Qualified exercise professional; BCS: Breast cancer survivor; HRQOL: Health related quality of Ifie; RCT: Randomized controlled trial; MVPA: Moderate-vigorous physical activity; APIM: Actor partner interdependence models; P1: Peer 1; P2: Peer 2; B: Beta; N: Number; SSS: Social support survey; SF-12: Short form-12; EQ-5D-3L: EuroQOL-5 dimension-3 level;WAI-SR: Work alliance inventory-short form revised.

\section{Supplementary Information}

The online version contains supplementary material available at https://doi. org/10.1186/s13102-021-00341-w.

Additional file 1. Outcome: Social support survey.

Additional file 2. Outcome: Use of health care services questionnaire.

Additional file 3. Outcome: Exercise peer-match quality quesitonnaire.

\section{Acknowledgements}

Not applicable.

Authors' contributions

All authors (JST, MM1, EO, AF, MM2, DSM, KAN, LT, JJ, JB, NCR, JT, MV, CS) were involved in the development of the study protocol. JST, EO, MM1, and CS drafted this manuscript and all other authors (MM2, AF, DSM, KAN, LT, JJ, JB, NCR, JT, MV) reviewed, provided feedback, and approved the manuscript for submission. All authors read and approved the final manuscript.

Funding

This work was supported by the Canadian Cancer Society Innovation to Impact Grant (grant \#: 507491). Independent peer-review took place during the funding process. The funding body had no role in the study design or writing of this manuscript.

Availability of data and materials

Not applicable.

\section{Declarations}

Ethics approval and consent to participate

The University of Toronto's Human Research Ethics Unit has approved this study (protocol \#00038665). All eligible participants will provide written informed consent prior to study enrollment.

Consent for publication

Not applicable.

Competing interests

The authors declare that they have no competing interests.

Author details

${ }^{1}$ School of Rehabilitation Science, McMaster University, 1400 Main Street West, Hamilton, ON L8S 1C7, Canada. ${ }^{2}$ Faculty of Kinesiology and Physical 
Education, University of Toronto, Toronto, Canada. ${ }^{3}$ Department of Kinesiology and Health, Rutgers University, New Brunswick, USA. ${ }^{4}$ Faculty of Kinesiology, University of Calgary, Calgary, Canada. ${ }^{5}$ Princess Margaret Cancer Centre, University Health Network, Toronto, Canada. ${ }^{6}$ School of Kinesiology and Health Studies, Queen's University, Kingston, Canada.

Received: 6 August 2021 Accepted: 8 September 2021 Published online: 14 October 2021

\section{References}

1. Canadian Cancer Society's Advisory Committee on Cancer Statistics. Canadian cancer statistics 2019. Toronto, ON: Canadian Cancer Society. 2020. Cancer.ca/Canadian-Cancer-Statistics-2019-EN

2. Bloom JR, Stewart SL, Oakley-Girvan I, Banks PJ, Shema S. Quality of life of younger breast cancer survivors: persistence of problems and sense of well-being. Psychooncology. 2012;21(6):655-65.

3. McFarland DC, Shaffer KM, Tiersten A, Holland J. Prevalence of physical problems detected by the distress thermometer and problem list in patients with breast cancer. Psychooncology. 2018;27(5):1394-403.

4. Mols F, Vingerhoets AJ, Coebergh JW, van de Poll-Franse LV. Quality of life among long-term breast cancer survivors: a systematic review. Eur J Cancer. 2005:41(17):2613-9.

5. Sabiston CM, Vallance J, Brunet J. Physical activity and cancer survivorship. In: Handbook of lifestyle medicine (2nd edn ). USA: Taylor \& Francis Inc; 2013.

6. Cheema BS, Kilbreath SL, Fahey PP, Delaney GP, Atlantis E. Safety and efficacy of progressive resistance training in breast cancer: a systematic review and meta-analysis. Breast Cancer Res Treat. 2014;148(2):249-68.

7. Kim J, Choi WJ, Jeong SH. The effects of physical activity on breast cancer survivors after diagnosis. J Cancer Prev. 2013;18(3):193-200.

8. Lee J, Lee MG. Effects of exercise interventions on breast cancer patients during adjuvant therapy: a systematic review and meta-analysis of randomized controlled trials. Cancer Nurs. 2020:43(2):115-25.

9. Ligibel JA, Giobbie-Hurder A, Shockro L, Campbell N, Partridge AH, Tolaney SM, et al. Randomized trial of a physical activity intervention in women with metastatic breast cancer. Cancer. 2016;122(8):1169-77.

10. McNeely ML, Campbell KL, Rowe BH, Klassen TP, Mackey JR, Courneya KS. Effects of exercise on breast cancer patients and survivors: a systematic review and meta-analysis. CMAJ. 2006;175(1):34-41.

11. Sabiston CM, Brunet J. Reviewing the benefits of physical activity during cancer survivorship. AJLM. 2012;6(2):167-77.

12. Schmid D, Leitzmann MF. Association between physical activity and mortality among breast cancer and colorectal cancer survivors: a systematic review and meta-analysis. Ann Oncol. 2014;25(7):1293-311.

13. Cannioto RA, Hutson A, Dighe S, McCann W, McCann SE, Zirpoli GR, et al. Physical activity before, during and after chemotherapy for high-risk breast cancer: relationships with survival. J Natl Cancer Inst. 2021;113(1):54-63.

14. Cormie P, Zopf EM, Zhang X, Schmitz K. The impact of exercise on cancer mortality, recurrence, and treatment-related adverse effects. Epidemiol Rev. 2017:39(1):71-92.

15. Ramírez-Vélez R, Zambom-Ferraresi F, García-Hermoso A, Kievisiene J, Rauckiene AR, Agostinis-Sobrinho C. Evidence-based exercise recommendations to improve mental wellbeing in women with breast cancer during active treatment: a systematic review and meta-analysis. Cancers (Basel). 2021;13(2):264

16. Campbell K, Winters-Stone K, Wiskemann J, May AM, Schwartz AL, Courneya KS, et al. Exercise guidelines for cancer survivors: consensus statement from international multidisciplinary roundtable. Med Sci Sports Exerc. 2019;51(11):2375.

17. Schmitz KH, Courneya KS, Matthews C, Demark-Wahnefried W, Galvao DA, Pinto BM, et al. American college of sports medicine roundtable on exercise guidelines for cancer survivors. Med Sci Sports Exerc. 2010:42(7):1409-26

18. Segal R, Zwaal C, Green E, Tomasone JR, Loblaw A, Petrella T, et al. Exercise for people with cancer: a clinical practice guidelines. Curr Oncol. 2017;24(1):40-6.

19. Blanchard CM, Courneya KS, Stein K. Cancer survivors' adherence to lifestyle behavior recommendations and associations with health-related quality of life: results from the American Cancer Society's SCS-II. J Clin Oncol. 2008;26(13):2198-204.

20. Fernandez S, Franklin J, Amlani N, DeMilleVille C, Lawson D, Smith J. Physical activity and cancer: a cross-sectional study on the barriers and facilitators to exercise during cancer treatment. Can Oncol Nurs 2015;42(1):37-48

21. Lynch BM, Dunstan DW, Healy GN, Winkler E, Eakin E, Owen N. Objectively measured physical activity and sedentary time of breast cancer survivors, and associations with adiposity: findings from NHANES (2003-2006). Cancer Causes Control. 2010;21(2):283-8.

22. Schmidt ME, Wiskemann J, Ulrich CM, Schneeweiss A, Steindorf K. Selfreported physical activity behavior of breast cancer survivors during and after adjuvant therapy: 12 months follow-up of two randomized exercise intervention trials. Acta Oncol. 2017;56(4):618-27.

23. Brunet J, Taran S, Burke S, Sabiston C. A qualitative exploration of barriers and motivators to physical activity participation in women treated for breast cancer. Disabil Rehabil. 2013;35(24):2038-45.

24. Sander AP, Wilson J, Izzo N, Mountford SA, Hayes KW. Factors that affect decisions about physical activity and exercise in survivors of breast cancer: a qualitative study. Phys Ther. 2011:92(4):525-36.

25. Smith-Turchyn J, Vani M, Sabiston C. Understanding how to reach the hard to reach in cancer rehabilitation. Glob J Nurs. 2020. https://doi.org/ 10.28933/gjn-2020-10-3005.

26. Wurz A, St-Aubin A, Brunet J. Breast cancer survivors' barriers and motives for participating in a group-based physical activity program offered in the community. Support Care Canc. 2015;23(8):2407-16.

27. Barber FD. Social support and physical activity engagement by cancer survivors. Clin J Oncol Nurs. 2012;16(3):E84-98.

28. Kampshoff CS, Stacey F, Short CE, van Mechelen W, Chinapaw MJ, Brug J, et al. Demographic, clinical, psychosocial, and environmental correlates of objectively assessed physical activity among breast cancer survivors. Support Care Canc. 2016;24(8):3333-42.

29. Phillips SM, McAuley E. Social cognitive influences on physical activity participation in long-term breast cancer survivors. Psychooncology. 2013;22(4):783-91.

30. McDonough MH, Beselt LJ, Daun JT, Shank J, Culos-Reed SN, Krounlund $\mathrm{LJ}$, et al. The role of social support in physical activity for cancer survivors: a systematic review. Psychooncology. 2019;28(10):1945-58.

31. Fong AJ, Scarapicchia TMF, McDonough MH, Wrosch C, Sabiston CM. Changes in social support predict emotional well-being in breast cancer survivors. Psychooncology. 2017;26(5):664-71.

32. Sabiston CM, McDonough MH, Crocker PRE. Psychosocial experiences of breast cancer survivors involved in a dragon boat program: exploring links to positive psychological growth. J Sport Exerc Psychol. 2007;29:419-38.

33. Dennis CL. Peer support within a health care context: a concept analysis. Int J Nurs Stud. 2003;40(3):321-32.

34. Castro CM, Pruitt LA, Buman MP, King AC. Physical activity program delivery by professionals versus volunteers: the TEAM randomized trial. Health Psychol. 2011;30(3):285-94.

35. Ginis KAM, Nigg CR, Smith AL. Peer-delivered physical activity interventions: an overlooked opportunity for physical activity promotion. Transl Behav Med. 2013·3(4):434-43.

36. DeMello MM, Pinto BM, Mitchell S, Dunsiger SI, Stein K. Peer support for physical activity adoption among breast cancer survivors: do the helped resemble the helpers? Eur J Cancer. 2018;27(3):e12849.

37. Pinto BM, Stein K, Dunsiger S. Peers promoting physical activity among breast cancer survivors: a randomized controlled trial. Health Psychol. 2015;34(5):463-72.

38. Fong AJ. Mind the (knowledge-to-action) gap: Exploring factors that affect physical activity in breast cancer survivors [Unpublished doctoral dissertation]. University of Toronto; 2017.

39. Smith-Turchyn J, Richardson J, Tozer R, Thabane R, McNeely M. Physical activity and breast cancer: results of a focus group to devise novel exercise interventions for women with breast cancer. Clin Nurs Stud. 2018:6(2):39-46.

40. Scott JM, Zabor EC, Schwitzer E, Kowlwyn GJ, Adams SC, Nilsen TS, et al. Efficacy of exercise therapy on cardiorespiratory fitness in patients with cancer: a systematic review and meta-analysis. J Clin Oncol. 2018;36(22):2297-305. 
41. Cook WL, Kenny DA. The actor-partner interdependence model: a model of bidirectional effects in developmental studies. Int J Behav Dev. 2005;29:101-9.

42. Kenny DA. Models of nonindependence in dyadic research. J Soc Pers Relat. 1996;13:279-94.

43. Kenny DA, Kashy DA, Cook WL. Dyadic data analysis. New York: Guilford; 2006

44. Courneya KS, Segal RJ, Gelmon K, Mackey JR, Friedenreich CM, Yasui Y, et al. Predictors of adherence to different types and doses of supervised exercise during breast cancer chemotherapy. Int J Behav Nutr Phys. 2014;11:85.

45. Leach HJ, Danyluk JM, Nishimura KC, Culos-Reed SN. Evaluation of a community based exercise program for breast cancer patients undergoing treatment. Cancer Nurs. 2015;38(6):417.

46. Schulz KF, Altman DG, Moher D. CONSORT 2010 statement: updated guidelines for reporting parallel group haracteri trials. J Pharmacol Pharmacother. 2010;1(2):100-7.

47. Chan AW, Tetzlaff JM, Altman DG, Laupacis A, Gotzsche PC, Krleza-Jeric K, et al. SPIRIT 2013 statement: defining standard protocol items for clinical trials. Ann Intern Med. 2013;158:200-7.

48. Ackerman RA, Kenny DA. APIMPowerR: an interactive tool for actor-partner interdependence model power analysis [Computer software]; 2016. https://robert-a-ackerman.shinyapps.io/APIMPowerRdis/.

49. Goodman JS, Blum TC. Assessing the non-random sampling effects of subject attrition in longitudinal research. J Manag. 1996;22(4):627-52.

50. Sabiston CM, Amireault S, Tamminen K, Fong A, Jones J. Exercise 'dating': instigating preferred social support for exercise through a match-making website for women cancer survivors. JSEP. 2015;37(Suppl):S142.

51. Sabiston C, Santa Mina D, Trihn L et al. Connecting breast cancer survivors for exercise: extending the impact of ActiveMatch. Canadian Cancer Society Research Institute Innovation to Impact Grant. 2019. https://webap ps.cihr-irsc.gc.ca/funding/detail_e?pResearchld=9091717\&p_version= CCS\&p_language $=E \& p \_s e s s i o n \_i d=3375057$.

52. Michie S, Ashford S, Sniehotta FF, Dombrowski SU, Bishop A, French DP. A refined taxonomy of behaviour change techniques to help people change their physical activity and healthy eating behaviours: the CALORE taxonomy. Psychol Health. 2011;26(11):1479-98.

53. Michie S, van Stralen MM, West R. The behaviour change wheel: a new method for haracterizing and designing behaviour change interventions. Implement Sci. 2011;6:42.

54. Trinh L, Arbour-Nicitopoulos K, Sabiston C, Berry SR, Loblaw A, Alibhai $\mathrm{SMH}$, et al. RiseTx: testing the feasibility of a web application for reducing sedentary behavior among prostate cancer survivors receiving androgen deprivation therapy. Int J Behav Nutr Phys Act. 2018;15(1):49.

55. University of Toronto. MoveUHappyU. https://www.moveu.ca/moveu happyu.

56. Amireault S, Godin G. The Godin-Shephard leisure-time physical activity questionnaire: validity evidence supporting its use for classifying healthy adults into active and insufficiently active categories. Percept Mot Skills. 2015;120(2):604-22.

57. Amireault S, Godin G, Lacombe J, Sabiston CM. The use of the GodinShephard leisure-time physical activity questionnaire in oncology research: a systematic review. BMC Med Res Methodol. 2015;15:60.

58. Godin G, Shephard RJ. A simple method to assess exercise behavior in the community. Can J Appl Sport Sci. 1985;10(3):141-6.

59. Hardcastle SJ, Jiménez-Castuera R, Maxwell-Smith C, Bulsara MK, Hince D. Fitbit wear-time and patterns of activity in cancer survivors throughout a physical activity intervention and follow-up: exploratory analysis from a randomised controlled trial. PLoS ONE. 2020;15(10):e0240967.

60. Rossi A, Frechette L, Miller D, Miller E, Friel C, Van Arsdale A, et al. Acceptability and feasibility of a Fitbit physical activity monitor for endometrial cancer survivors. Gynecol Oncol. 2018;149(3):470-5.

61. Rees T, Hardy L, Evans L. Construct validity of the social support survey in sport. Psychol Sport Exerc. 2007;8(3):355-68.

62. Richman JM, Rosenfeld LB, Hardy CJ. The social support survey: a validation study of a clinical measure of the social support process. Res Soc Work Pract. 1993;3(3):288-311.
63. McDonough MH, Sabiston CM, Wrosch C. Predicting changes in posttraumatic growth and subjective well-being among breast cancer survivors: the role of social support and stress. Psychooncology. 2014;23(1):114-20.

64. Gandek B, Ware JE, Aaronson NK, Apolone G, Bjorner JB, Brazier JE, et al. Cross-validation of item selection and scoring for the SF-12 health survey in nine countries: results from the IQOLA project. International quality of life assessment. J Clin Epidemiol. 1998;51(11):1171-8.

65. Ware JJ, Kosinski M, Keller SD. A 12-item short-form health survey: construction of scales and preliminary tests of reliability and validity. Med Care. 1996;34(3):220-33.

66. EuroQOL Group. EQ-5D-3L user guide: basic information on how to use the EQ-5D-3L instrument. Version 5.1. 2015. https://euroqol.org/wpcontent/uploads/2016/09/EQ-5D-3L_UserGuide_2015.pdf.

67. Simon Pickard A, Wilke C, Lin H, Lloyd A. Health utilities using the EQ-5D in studies of cancer. Pharmacoeconomics. 2007;25(5):365-84

68. Richardson J, Letts L, Chan D, Officer A, Wojkowski S, Oliver D, et al. Monitoring physical functioning as the sixth vital sign: evaluating patient and practice engagement in chronic illness care in a primary care setting - a quasi-experimental design. BMC Fam Pract. 2012;13:29.

69. Rosenberg DE, Norman GJ, Wagner N, Patrick K, Calfas KJ, Sallis JF. Reliability and validity of the sedentary behavior questionnaire (SBQ) for adults. J Phys Act Health. 2010;7(6):697-705.

70. Swain CTV, Nguyen NH, Eagles T, Vallance JK, Boyle T, Lahart IM, et al. Postdiagnosis sedentary behavior and health outcomes in cancer survivors: a systematic review and meta-analysis. Cancer. 2020;126(4):861-9.

71. Paap D, Dijkstra PU. Working alliance inventory-short form revised. J Physiother. 2017;63(2):118.

72. Warburton DER, Jamnik VK, Bredin SSD, Gledhill N. The 2014 physical activity readiness questionnaire for everyone (PAR-Q+) and electronic physical activity readiness medical examination (ePARmed-X+). HFJC. 2014;7(1):80-3.

73. The R Foundation. The R project for statistical computing. 2021. https:// www.r-project.org

74. MPLUS Version 7.4 (Muthén \& Muthén, Los Angeles, CA).

75. Sabiston CM, Fong AJ, O'Loughlin EK, Meterissian S. A mixed-methods evaluation of a community physical activity program for breast cancer survivors. J Transl Med. 2019;17(1):206.

76. MacDonald AM, Chafranskaia A, Lopez CJ, Maganti M, Bernstein LJ, Change E, et al. CaRE @ Home: pilot study on an online multidimensional cancer rehabilitation and exercise program for cancer survivors. J Clin Med. 2020;9(10):3092.

77. Cheifetz O, Park Dorsay J, Hladysh G, Macdermid J, Serediuk F, Woodhouse LJ. CanWell: meeting the psychosocial and exercise needs of cancer survivors by translating evidence into practice. Psychooncology. 2014;23(2):204-15.

78. McNeely M, Sellar C, Williamson T, Shea-Budgell M, Abraham Joy A, Lau $H Y$, et al. Community-based exercise for health promotion and secondary cancer prevention in Canada: protocol for a hybrid effectiveness-implementation study. BMJ Open. 2019;9:e029975.

79. Santa Mina D, Sabiston CM, Au D, Fong AJ, Capozzi LC, Langelier D, et al. Connecting people with cancer to physical activity and exercise programs: a pathway to create accessibility and engagement. Curr Oncol. 2018;25(2):149-62.

80. Shaghaghi A. Approaches to recruiting 'hard-to-reach' populations into research: a review of the literature. Health Promot Perspect. 2011;1(2):86-94.

81. Barnidge EK, Radvanyi C, Duggan K, Motton F, Wiggs I, Baker EA, et al. Understanding and addressing barriers to implementation of environmental and policy interventions to support physical activity and healthy eating in rural communities. J Rural Health. 2013;29(1):97-105.

82. Cleland V, Hughes C, Thornton L, Venn A, Squibb K, Ball K. A qualitative study of environmental factors important for physical activity in rural adults. PLOS ONE. 2015;10(11):e0140659.

83. Wu YP, Yi J, McClellan J, Kim J, Tian T, Grahmann B, et al. Barriers and facilitators of healthy diet and exercise among adolescent and young adult cancer survivors: implications for behavioral interventions. J Adolesc Young Adult Oncol. 2015;4(4):184-91. 
84. McEachan RR, Conner M, Taylor NT, Lawton RJ. Prospective prediction of health- related behaviours with the theory of planned behaviour: a metaanalysis. Health Psychol Rev. 2011;5(2):97-144.

85. Newton RU, Hart NH, Clay T. Keeping patients with cancer exercising in the age of COVID-19. J Oncol Pract. 2021;16(10):656-64

86. Galiano-Castillo N, Cantarero-Villanueva I, Fernandez-Lao C, ArizaGarcia A, Diaz-Rodriguez L, Del-Moral-Avila R, et al. Telehealth system: a randomized controlled trial evaluating the impact of an internet-based exercise intervention on quality of life, pain, muscle strength, and fatigue in breast cancer survivors. Cancer. 2016;122:3166-74.

87. Buneviciene I, Mekary R, Smith T, Onnela JP, Bunevicius A. Can mHealth interventions improve quality of life of cancer patients? A systematic review and meta-analysis. Crit Rev Oncol Hematol. 2021;157:103123.
88. Post KE, Flanagan J. Web based survivorship interventions for women with breast cancer: an integrative review. Eur J Oncol Nurs. 2016;25:90-9.

89. Adams SC, Smith-Turchyn J, Santa Mina D, et al. The exercise oncology knowledge mobilization initiative (ExOnc-KMI): an international modified delphi study. Front Oncol. 2021;11:713199.

\section{Publisher's Note}

Springer Nature remains neutral with regard to jurisdictional claims in published maps and institutional affiliations.
Ready to submit your research? Choose BMC and benefit from:

- fast, convenient online submission

- thorough peer review by experienced researchers in your field

- rapid publication on acceptance

- support for research data, including large and complex data types

- gold Open Access which fosters wider collaboration and increased citations

- maximum visibility for your research: over $100 \mathrm{M}$ website views per year

At BMC, research is always in progress.

Learn more biomedcentral.com/submissions 